\title{
Efectos del Curado en las Propiedades de Mezclas de Concreto con Altos Contenidos de Escoria de Hierro
}

\author{
Juan M. Lizarazo(1), Andres Salas(2), Diego A. Escobar ${ }^{(2)}$ \\ (1) Facultad de Ingeniería, Dpto. de Ingeniería Civil y Agrícola, Univ. Nacional de Colombia, Bogotá - \\ Colombia. (e-mail: jmlizarazom@unal.edu.co) \\ (2) Facultad de Ingeniería y Arquitectura, Dpto. de Ingeniería Civil, Univ. Nacional de Colombia, Manizales - \\ Colombia. (e-mail: asalasmo@unal.edu.co, daescobarga@unal.edu.co)
}

Recibido Feb. 10, 2016; Aceptado Mar. 28, 2016; Versión final Abr. 19, 2016, Publicado Dic. 2016

\section{Resumen}

Se determinaron las propiedades de resistencia a la compresión, resistencia a la carbonatación y resistencia a la penetración de ion cloruro en mezclas de concreto adicionadas con altos volúmenes de escoria granulada de alto horno (GGBS, por sus siglas en inglés) con relaciones agua cemento de 0.4 y 0.5 . Adicionalmente, en el estudio se investigó el efecto del curado en las propiedades mencionadas. Se evaluaron dos métodos de curado: (1) curado al aire y (2) curado húmedo. Los resultados mostraron que las condiciones de curado seco tienen un efecto adverso en las propiedades de mezclas, especialmente de aquellas con altos contenidos de escorias. En contraste, las muestras curadas bajo condiciones de humedad presentaron un desempeño igual o superior al de las mezclas producidas únicamente con cemento Portland común.

Palabras clave: concretos de alto desempeño; materiales cementantes alternativos; escoria granulada de alto horno; resistencia de concreto

\section{Effects of Curing on Properties of Concrete Mixtures with High Iron-Slag Content}

\begin{abstract}
In this paper, the compressive strength, the resistance to carbonation and the chloride ion penetration of concrete mixes blended with high volume ground granulated blast furnace slag (GGBS) with water-to-binder ratios of 0.4 and 0.5 were determined. The study also investigated the effect of curing on the mentioned properties. Two methods of curing were evaluated: (1) air-dry curing, and (2) wet curing. The results showed that the dry curing conditions have an adverse effect on the properties studied, especially at high volume of GGBS. In contrast, wet-cured blended samples showed similar or even superior performance than the mixes using only the common Portland cement.
\end{abstract}

Keywords: high performance concretes; supplementary cementing materials; ground granulated blast furnace slag; concrete resistance 


\section{INTRODUCCIÓN}

La escoria siderúrgica es un subproducto del proceso de fabricación de metales férreos. Sideros significa hierro en lengua griega clásica (Gaud, 2013). Dependiendo del tipo de metales producidos, las escorias tendrán diferentes propiedades mineralógicas. La producción de hierro, acero, cobre, plomo y aluminio dominan la industria metalúrgica, generando grandes volúmenes de escoria. Dependiendo de sus propiedades físicas y químicas una escoria puede ser usada en la industria de la construcción como agregado en bases granulares, mezclas de asfalto, como cementante y como reemplazo del cemento Portland en mezclas de concreto, y puede ser usada en aplicaciones a granel como en terraplenes y rellenos. El uso de escoria como material cementante o como reemplazo en mezclas de concreto ha sido extensamente estudiado por diversas razones como: los beneficios ambientales, sus propiedades cementantes o puzolánicas potenciales y el alto costo del cemento Portland a nivel mundial. La escoria más comúnmente utilizada es la escoria granulada de alto horno (GGBS, por sus siglas en inglés), producida como desecho de la industria manufacturera del hierro (Qingtao et al., 2012; Chidiac y Panesar, 2008; Yazici et al., 2010; Hadjsadok et al., 2012; Elkhadiri et al.,2009).

El curado tiene como objetivo la preservación de un aceptable contenido de humedad y temperatura en el concreto. Durante el proceso de hidratación, una serie de reacciones químicas toman lugar entre el cemento Portland y el agua de mezclado. La resistencia del concreto y su durabilidad son variables fuertemente influenciadas por el porcentaje en que estas reacciones químicas se produzcan. Es muy bien conocido que el curado tiene una fuerte influencia sobre las propiedades del concreto endurecido (Çakir y Aköz, 2008; Nassif et al., 2005; Al-Gahtani, 2010, Escalante et al, 2001). Sin embargo, los efectos del medio de curado sobre la hidratación de cementos mezclados requiere mayor investigación, se hace necesario evaluar la hidratación de la escoria granulada de alto horno en cementos adicionados bajo diferentes condiciones de curado, con el ánimo de estimar las propiedades y la durabilidad de las estructuras de estos concretos.

Hasta ahora, estos efectos se han medido por varios investigadores bajo condiciones de laboratorio, que representan un caso ideal, pero con el ánimo de representar ambientes más realistas, en la presente investigación se ha considerado comparar el efecto de condiciones de curado al aire libre y bajo condiciones controladas de laboratorio. A pesar de que las propiedades mecánicas y la resistencia a la carbonatación de mezclas de concreto adicionadas con GGBS son bien conocidas, su resistencia a la carbonatación se encuentra aún en debate (Sulapa et al, 2003; Jia et al, 2012), adicionalmente, se ha sugerido que el efecto del tipo y calidad del curado son factores determinantes en las mezclas que contienen altos volúmenes de GGBS (Lee, 2008), lo que puede ser determinante en aplicaciones reales de la construcción que realizan comúnmente un pobre curado. Tomando en cuentas las consideraciones anteriores, el objetivo principal del presente artículo es el de estudiar la resistencia a la compresión, la resistencia a la carbonatación y a la penetración del ion cloruro en mezclas de concreto con altos volúmenes de GGBS sometidas a diferentes condiciones de curado: bajo condiciones controladas de laboratorio y al aire bajo un ambiente seco. Para esto se prepararon dos tipos de concretos con diferentes relaciones agua cemento y se llevaron a cabo ensayos experimentales estandarizados para su caracterización.

\section{MATERIALES Y MÉTODOS}

Se describen los materiales usados, su composición química, mineralogía, propiedades físicas: Luego se explica cómo se preparan las mezclas, y se explican después los procedimientos de ensayo y medición de la resistencia a la compresión, la resistencia a la carbonatación, y la penetración de iones cloruro.

\section{Materiales utilizados}

Materiales Cementantes: Se utilizó Cemento Portland Ordinario (CPO) con 95-100\% de Clinker, clasificado como CEM-1 de acuerdo a la norma Británica BS EN 197-1 (2011). Este cemento se encontraba libre de adiciones excepto por el yeso incorporado. Escoria granulada de alto horno, disponible en el comercio del Reino Unido, se utilizó como material puzolánico de referencia para esta investigación. El material se presenta bajo la norma BS 6699 "Especificación para Escoria Granulada de Alto Horno para uso con cemento Portland". A continuación se presentan los resultados de la caracterización de los materiales cementantes utilizados.

Composición Química: La tabla 1 presenta los análisis químicos tanto del cemento Portland ordinario, como de la Escoria granulada de alto horno. Para la GGBS, los valores medidos para el residuo insoluble, magnesia, sulfuro y pérdida al fuego, se encuentran dentro de los límites especificados por la norma BS 15167 (2006). Adicionalmente, los requerimientos del mismo estándar para la composición de $\mathrm{CaO}, \mathrm{MgO}$, y $\mathrm{SiO} 2$, se cumplieron plenamente. 
Tabla 1: Composición química de los materiales cementantes utilizados

\begin{tabular}{|c|c|c|c|c|c|c|c|c|c|c|c|c|}
\hline & \multicolumn{10}{|c|}{ Composición química [\%] } \\
\hline Cementante & $\mathrm{SiO}_{2}$ & $\mathrm{TiO}_{2}$ & $\mathrm{Al}_{2} \mathrm{O}_{3}$ & $\mathrm{Fe}_{2} \mathrm{O}_{3}$ & $\mathrm{MnO}$ & $\mathrm{MgO}$ & $\mathrm{CaO}$ & $\mathrm{Na}_{2} \mathrm{O}$ & $\mathrm{K}_{2} \mathrm{O}$ & $\mathrm{P}_{2} \mathrm{O}_{5}$ & $\mathrm{SO}_{3}$ & $\mathrm{PF}$ \\
\hline $\mathrm{CPO}$ & 19.7 & --- & 4.9 & 2.4 & --- & 2.1 & 63.3 & 0.2 & 0.6 & -- & 2.7 & 2.7 \\
\hline GGBS & 34.5 & 0.55 & 13.16 & 0.74 & 0.45 & 7.75 & 38.7 & 0.29 & 0.55 & 0.02 & 1.75 & 0.7 \\
\hline
\end{tabular}

Mineralogía: La figura 1 muestra los patrones de difracción de rayos $\mathrm{X}$ obtenidos para los materiales cementantes. Las fases principales que se pueden identificar en el cemento Portland son $\mathrm{C}_{3} \mathrm{~S}, \mathrm{C}_{2} \mathrm{~S}, \mathrm{C}_{3} \mathrm{~A}, \mathrm{y}$ $\mathrm{C}_{4} \mathrm{AF}$, adicionalmente se pueden encontrar algunos picos correspondientes a carbonato de calcio (calcita), (Chatterjee 2001). Los patrones de difracción de la GGBS muestran una banda difusa donde se diferencian la merwinita y la melilita, como se reportó en Taylor (1990).
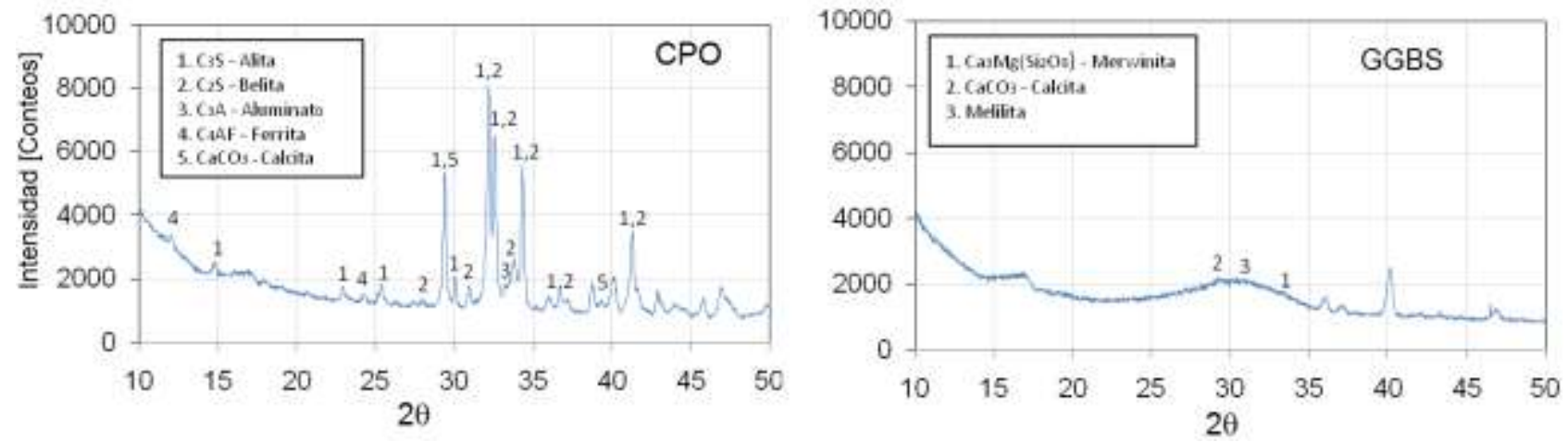

Fig. 1: Análisis de difracción de rayos X para el CPO y la GGBS (adaptada de Lizarazo, 2010)

Propiedades Físicas: Los pesos específicos (densidades) del CPO y de la GGBS fueron de $3110 \mathrm{~kg} / \mathrm{m}^{3}$ y de $2940 \mathrm{~kg} / \mathrm{m}^{3}$ respectivamente. La Figura 2 presenta la distribución de tamaño y las forma de las partículas de los dos materiales cementantes, obtenidas con un equipo de granulometría láser, en el que se utilizó como agente dispersante metafosfato de sodio. Como aditivo superplastificante se usó un policarboxilato de tipo comercial
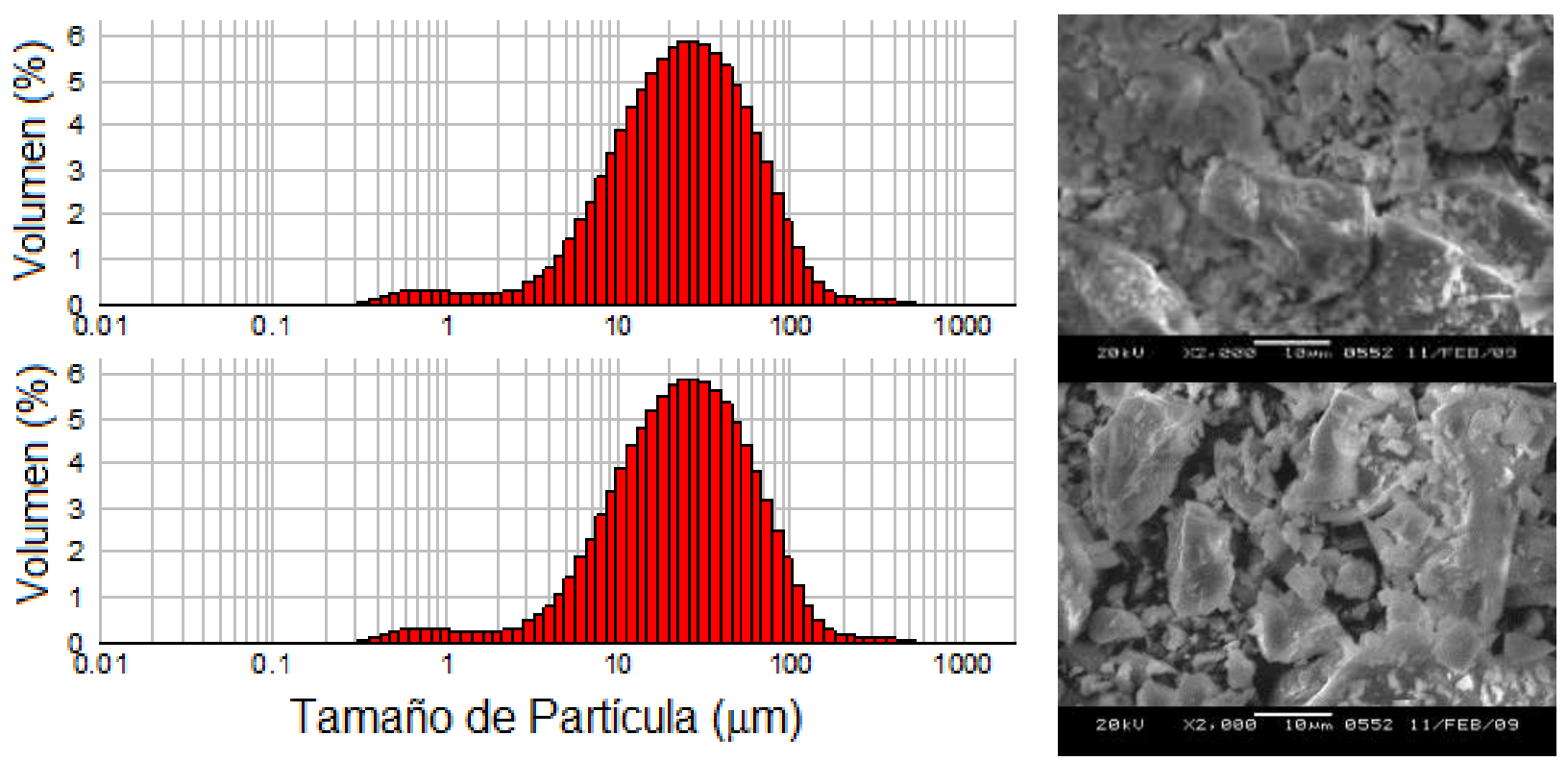

Fig. 2: Distribución de tamaño y forma de las partículas de los materiales cementantes (adaptada de Lizarazo, 2010).

Agregados: Un agregado grueso de cantera de origen sedimentario, con partículas de superficie mixta entre rugosa y redondeada, con una ligera falta de finos de tamaño inferior a 0,3 $\mathrm{mm}$, se utilizó para las mezclas de concreto, con un tamaño máximo nominal de $13 \mathrm{~mm}$. La Figura 3 muestra la distribución de tamaños de las partículas del agregado y los límites recomendados en la norma ASTM C33 (ASTM, 2013). La granulometría del material se encontró entre los límites del tamaño número 7 de acuerdo a la norma ASTM. Como agregado fino se utilizó arena silícea de rio. El análisis granulométrico de la arena se muestra en la 
figura 3, donde se incluyen también los límites establecidos en la norma ASTM C33, se puede observar que el material se encuentra cerca del límite inferior siendo una arena gruesa. El módulo de finura calculado fue de 3.3 y la densidad seca fue de $2,660 \mathrm{~kg} / \mathrm{m}^{3}$. La gravedad específica promedio del cemento utilizado fue de 3.04. En la figura 3 se presenta un gráfico de la granulometría completa de la mezcla de áridos junto con la curva de Fuller correspondiente a su tamaño máximo.
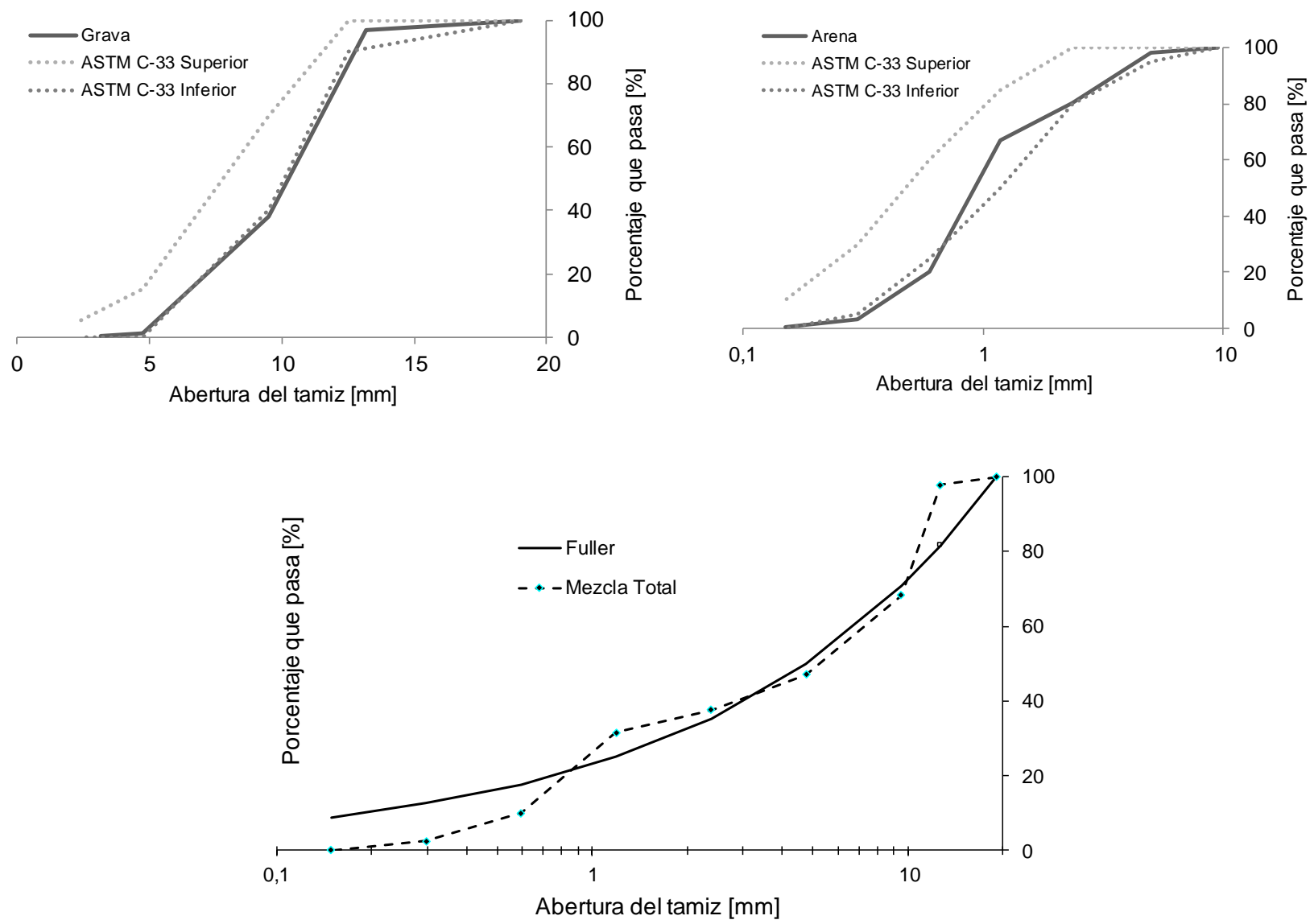

Fig. 3: Distribución de tamaños de los agregados con los límites de la norma ASTM C-33 y comparación de la combinación con la granulometría especificada por Fuller (adaptada de Lizarazo, 2010).

\section{Diseños de Mezcla}

Para el desarrollo de la presente investigación se utilizaron mezclas con altos volúmenes de GGBS usando $60 \%$ como reemplazo de cemento Portland y mezclas patrón con $100 \%$ cemento Portland. Las mezclas se diseñaron con dos relaciones agua-material cementante de 0.40 y 0.50 . Las proporciones de los materiales en peso por metro cubico de concreto se muestran en la Tabla 2.

Tabla 2: Proporciones de las Mezclas de Concreto

\begin{tabular}{|c|c|c|c|c|c|c|c|}
\hline \multirow{2}{*}{ Proporciones } & \multirow{2}{*}{ Mezcla } & \multirow{2}{*}{$\mathrm{a} / \mathrm{cm}$} & \multicolumn{3}{|c|}{ Aglutinante $\left(\mathrm{kg} / \mathrm{m}^{3}\right)$} & Arena & Grava \\
\cline { 4 - 8 } & & & CPO & GGBS & Total & $\mathrm{kg} / \mathrm{m}^{3}$ & $\mathrm{~kg} / \mathrm{m}^{3}$ \\
\hline $100 \%$ CPO (referencia) & O4 & 0.4 & 400 & 0 & 400 & 795 & 972 \\
\hline $100 \%$ CPO (referencia) & O5 & 0.5 & 400 & 0 & 400 & 747 & 913 \\
\hline $40 \%$ CPO+ 60\% GGBS & OG4 & 0.4 & 160 & 240 & 400 & 805 & 984 \\
\hline $40 \%$ CPO+ 60\% GGBS & OG5 & 0.5 & 160 & 240 & 400 & 757 & 925 \\
\hline
\end{tabular}

Mezclado y curado.

Las mezclas de concreto se prepararon utilizando una mezcladora mecánica horizontal de 50 litros de capacidad. El proceso de mezcla se realizó siguiendo lo establecido en la norma ASTM C192 (ASTM, 2012). El mezclado se inició incorporando el agregado grueso a la mezcladora junto con un tercio del agua de mezclado. El agregado grueso y el agua se mezclaron por 30 segundos para saturar los agregados. La 
mezcladora se detuvo para añadir los materiales cementantes y el agregado fino y se reinició el mezclado añadiendo lentamente el agua restante. Seguidamente se mezclaron los ingredientes por 3 minutos, después de los cuales se detuvo la mezcladora por 3 minutos y finalmente se mezclaron por 2 minutos. Cuando se usaron, los superplastificantes se incorporaron a la mezcla lentamente durante los primeros 3 minutos de mezclado. Con la mezcla preparada se llenaron los moldes, se compactaron y se vibraron, posteriormente se cubrieron con plásticos y permanecieron durante 24 horas a una temperatura de $20 \pm 3$ ${ }^{\circ} \mathrm{C}$, después de las cuales los especímenes se desmoldaron y se llevaron a curado hasta el día de ensayo. Para cada mezcla de concreto, los ensayos se llevaron a cabo en muestras sometidas a dos diferentes condiciones de curado. El curado húmedo, en el cual las mezclas se sumergieron en agua con cal y se mantuvieron a $20 \pm 2{ }^{\circ} \mathrm{C}$. En contraste, el curado seco, en el cual las muestras se dejaron al aire libre a $20 \pm 4$ ${ }^{\circ} \mathrm{C}$ y humedad relativa entre $40-60 \%$.

\section{PROCEDIMIENTOS DE ENSAYO}

En la Tabla 3 se resumen los experimentos llevados a cabo en el presente estudio, en el cual se evaluaron 4 mezclas de concreto bajo dos condiciones diferentes de curado. En total se investigaron 8 diferentes tipos de especímenes. Las muestras denominadas con la letra "W" se sumergieron en agua, mientras que las muestras marcadas con "A" se curaron al aire.

Resistencia a la Compresión. La resistencia a la compresión de las mezclas de concreto se obtuvo siguiendo las recomendaciones de la norma: European Standard EN 12390-3:2001: "Testing hardened concrete, Part 3: Compressive strength of test specimens", en especímenes cúbicos con $100 \mathrm{~mm}$ de tamaño. La resistencia a la compresión se midió a las edades de 3, 28, y 90 días.

Tabla 3: Experimentos realizados

\begin{tabular}{|c|c|c|c|c|c|c|}
\hline Proporciones & $\mathrm{a} / \mathrm{cm}$ & curado & muestra & $\begin{array}{c}\text { Especímenes } \\
\text { para Resistencia } \\
\text { a Compresión }\end{array}$ & $\begin{array}{c}\text { Especímenes } \\
\text { para } \\
\text { Carbonación }\end{array}$ & $\begin{array}{c}\text { Especímenes } \\
\text { para Penetración } \\
\text { Cloruros }\end{array}$ \\
\hline $100 \%$ CPO (referencia) & 0.40 & Húmedo & O4W & 3 & 3 & 3 \\
\hline $100 \%$ CPO (referencia) & 0.40 & aire & O4A & 3 & 3 & 3 \\
\hline $100 \%$ CPO (referencia) & 0.50 & Húmedo & O5W & 3 & 3 & 3 \\
\hline $100 \%$ CPO (referencia) & 0.50 & aire & O5A & 3 & 3 & 3 \\
\hline $40 \%$ CPO+ 60\% GGBS & 0.40 & Húmedo & OG4W & 3 & 3 & 3 \\
\hline $40 \%$ CPO+ 60\% GGBS & 0.40 & aire & OG4A & 3 & 3 & 3 \\
\hline $40 \%$ CPO+ 60\% GGBS & 0.50 & Húmedo & OG5W & 3 & 3 & -- \\
\hline $40 \%$ CPO+ 60\% GGBS & 0.50 & aire & OG5A & 3 & 3 & -- \\
\hline
\end{tabular}

Resistencia a la Carbonatación. La profundidad de carbonatación se midió en especímenes cúbicos a la edad de 1 año de curado. Durante los primeros 28 días los especímenes se curaron bajo las condiciones específicas previamente establecidas, secos o húmedos. Posteriormente las probetas se removieron de su ambiente de curado se mantuvieron bajo condiciones ambientales naturales en un ambiente protegido de la lluvia y de la nieve en la cubierta de un edificio de la Universidad de Coventry en Inglaterra hasta la fecha del ensayo. Las condiciones circundantes a la mezclas corresponden a las de una ciudad de tamaño mediano con cuatro estaciones. Durante ésta investigación, se usó el modelo de la raíz cuadrada para cuantificar los mecanismos de carbonatación (Ver expresión (1)). En este, la profundidad de carbonatación $\mathrm{x}_{\mathrm{c}}$ se relaciona con el tiempo de exposición $\mathrm{t}$, a través del coeficiente de carbonatación, $\mathrm{k}_{\mathrm{c}}$, el método para medir la profundidad de carbonatación fue el método de la fenolftaleína recomendado por el RILEM (TC56RILEM / CPC-18, 1988). Se esparce sobre una superficie de la muestra recientemente fracturada una solución de $1 \%$ de fenolftaleína en $70 \%$ de alcohol etílico. En la parte no carbonatada del espécimen, se obtiene un color purpura a los pocos minutos, mostrando que el concreto aún presenta alta alcalinidad. En contraste, en la parte carbonatada no existe coloración. Para el cálculo del coeficiente de carbonatación se usaron cubos de $100 \mathrm{~mm}$ de lado.

$x_{c}=k_{c} \sqrt{t}$

Penetración de lones Cloruro. Los ensayos de inmersión en cloruros se realizaron con el objeto de medir la penetración de los mismos en las muestras de concreto después de un proceso de difusión normal. Para todos los ensayos, los cilindros preparados (100 mm diámetro y $200 \mathrm{~mm}$ altura) se dividieron en dos mitades y las superficies cortadas (100 mm diámetro y $100 \mathrm{~mm}$ altura) se colocaron en contacto con una solución de cloruro de sodio. Para preparar las muestras, la superficie curva de cada cilindro se recubrió con 
una capa de caucho siliconado con el objeto de evitar el intercambio de humedad y la carbonatación durante el ensayo. Después del recubrimiento, la superficie despejada de las muestras se colocó en contacto con una solución de $0.5 \mathrm{~g} / \mathrm{l}$ of $\mathrm{CaOH}$ durante una semana para evitar los efectos de la sorción inicial cuando se introduce la solución de cloruros. La concentración externa de cloruros fue de $7 \% \mathrm{NaCl}$ preparada con agua destilada y se renovó periódicamente con el objeto de conservar las condiciones de borde. Durante el ensayo, la solución se agito regularmente.

Al final del periodo de inmersión se removieron los especímenes de concreto y se determinó la penetración de cloruros después de esparcir una solución de nitrato de plata sobre la muestra. Después de realizada la aspersión sobre la superficie cortada de la solución de nitrato de plata, se observó la precipitación de cloruro de plata blanco sobre la muestra, con la cual se pudo calcular la profundidad de penetración promedio. Los experimentos iniciaron 90 días después de la preparación de las muestras y se prolongaron por otros 90 días. Los especímenes se curaron de acurdo a las condiciones especificadas antes del inicio de los ensayos y tres replicas se ensayaron por cada mezcla. De acuerdo con Baroghel-Bouny et al. (2007), la técnica calorimétrica con nitrato de plata provee excelentes resultados cuando se necesita investigar el desempeño de un grupo de concretos expuestos a la misma concentración de cloruros. Se hace posible cuantificar, comparar y calificar las mezclas de acuerdo al coeficiente $k$ de la expresión (2):

$$
x_{d}=k \sqrt{t}
$$

\section{RESULTADOS Y DISCUSIÓN}

Resistencia a la compresión. La Figura 4 presenta los resultados de resistencia a la compresión para todas las mezclas evaluadas. La variabilidad de los ensayos se muestra a través de las barras de error con una amplitud igual a la desviación estándar. Se observaron las siguientes condiciones para todas las mezclas: A mayor edad de curado mayor resistencia, las muestras curadas en agua presentaron mayor resistencia a la compresión que sus equivalentes curadas al aire, y la reducción en la relación agua/cementante produjo un incremento en la resistencia.

Desarrollo resistente: La resistencia a la compresión a los 90 días se entenderá en la presente investigación como la resistencia final; sin embargo, esto no significa que a mayores edades no se puedan presentar incrementos en la misma. La mezcla O4W (CPO, a/cm=0.4, curada al agua) desarrolló a los 3 días el $60 \%$ y a los 28 días desarrollo el $91 \%$ de sus resistencia final. La mezcla OG4W presentó a las primeras edades un retraso en la velocidad de adquisición de resistencias, esta mezcla desarrolló un $37 \%$ a los 3 días y a los 28 días un $86 \%$ de su resistencia final. La GGBS produce un retardo a edades tempranas dado que los componentes vítreos de la escoria reaccionan lentamente con el agua y toman tiempo para desarrollar los productos de hidratación. Sin embargo, a mayores edades pueden producir mayores resistencias que las mezclas ordinarias con CPO.

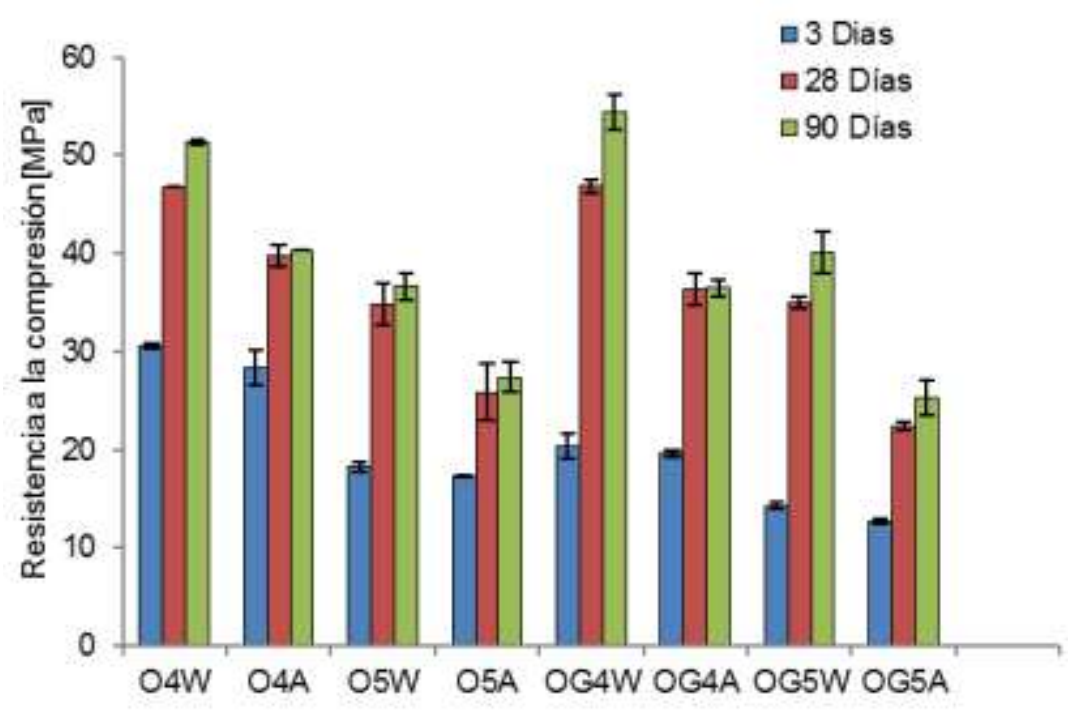

Fig. 4: Resistencia a la compresión de mezclas de concreto adicionadas (adaptada de Lizarazo, 2010).

Influencia del curado. Para todas las mezclas curadas aire, el porcentaje de resistencia a la compresión desarrollado para cualquier mezcla a los 28 días, con respecto al valor de la resistencia de la misma mezcla 
a los 90 días fue mucho mayor que cuando las muestras se curaron agua. Esta observación es importante porque significa que cuando las muestras no se curan adecuadamente (es decir, en el aire), estas no presentaran un aumento significativo en su resistencia a edades posteriores (después de 28 días). Los valores de resistencia observados a los 28 días y 90 días eran relativamente similares. Las reducciones en los resultados de resistencia a la compresión debido a las diferencias en el curado se observaron en todas las mezclas de concreto. Por ejemplo, la mezcla O4 presento una reducción del $4 \%$ a los 3 días, del $15 \%$ a los 28 días y del $21.5 \%$ a los 90 días cuando se curó de aire. De la misma manera, para la mezcla O5 la reducción fue del $5.5 \%$ a los 3 días, del $25.7 \%$ a los 28 días y de $25.2 \%$ a los 90 días. Para todas las mezclas a edades tempranas (3 días) se observó que el curado al aire no afectó significativamente la resistencia a la compresión. Esto podría deberse a que existe la cantidad de agua necesaria en las muestras para la hidratación de las mismas al menos durante los primeros días. Las diferencias en las condiciones de curado dieron como resultado una reducción de la resistencia a la compresión; a los 90 días, por ejemplo la reducción se puede observar en la Tabla 4.

Tabla 4: Reducción en la resistencia a la compresión a los 90 días debido al tipo de curado

\begin{tabular}{|c|c|c|c|c|}
\hline Mezcla & O4 & O5 & OG4 & OG5 \\
\hline Reducción [\%] & 21.5 & 25.2 & 33.0 & 37.0 \\
\hline
\end{tabular}

Influencia de la Relación Agua - Cementante. Para las mezclas de CPO a los 90 días, un incremento en la relación agua-cementante presenta un mayor impacto sobre la resistencia que el método de curado. En contraste, las mezclas CPO-GGBS son más susceptibles al método de curado que a la relación aguacementante. Para cualquier edad y tipo de curado, como era de esperarse, el factor más influyente en los la reducción de la resistencia a la compresión fue el cambio en la relación agua-cementante, siendo mayor en edades tempranas. Para todos los cementantes, la reducción de la resistencia debido a cambios en la relación agua-cementante fue mayor para las muestras curadas al aire. Para todas las mezclas, la Tabla 5 muestra la reducción en la resistencia a la compresión a los 90 días debido al incremento en la relación agua-cementante.

Tabla 5: Reducción de la resistencia a la compresión a los 90 días debido a la relación agua-cementante

\begin{tabular}{|c|c|c|c|c|}
\hline Mezcla & OW & OA & OGW & OGA \\
\hline Reducción [\%] & 28.5 & 31.9 & 26.2 & 30.7 \\
\hline
\end{tabular}

Efecto de los Materiales: A edades tempranas (3 días), independientemente del método de curado o de la relación agua-cementante, para las mezclas GGBS la resistencia a la compresión medida fue menor que la de las mezclas CPO de referencia. A los 90 días, la resistencia a la compresión de las muestras de CPOGGBS curadas bajo el agua fue mayor que la de referencia - se obtuvo un aumento entre el 6 y el $9.5 \%$ para las muestras con una relación agua-cementante de 0.4 y 0.5 respectivamente. Estos resultados indican que si la mezcla CPO-GGBS se cura adecuadamente bajo agua, se puede esperar que tenga una resistencia superior a la de la que contiene $100 \%$ de cemento Portland ordinario. Para la mezcla de GGBS curada al aire, se observó una reducción de $9.3 \%$ y $7.8 \%$ en comparación con la mezcla de referencia CPO y que se obtuvo para muestras con relaciones agua-cementante de 0.4 y 0.5 respectivamente.

Resistencia a la Carbonatación. Un año después de producidos, los especímenes se cortaron en dos mitades y se examinó la profundidad de carbonatación mediante el esparcimiento de una fina niebla de una solución acuosa de fenolftaleína en alcohol etílico utilizada como indicador de $\mathrm{pH}$ sobre la superficie fraccionada de los especímenes. Después de un par de minutos emergió una frontera bien definida, indicando 2 zonas distintas con valores marcadamente diferentes de $\mathrm{pH}$. Los resultados para la profundidad de carbonatación calculada con la expresión (1) se muestran en la Figura 5. Se observó que esta profundidad es principalmente una función del tipo de cementante, además, del régimen de curado, y por último, pero también importante, de la relación agua-cementante. Como ha indicado Basheer (2001), la carbonatación se relaciona con el coeficiente de difusión dióxido de carbono intrínseco del material y con la reserva de hidróxido de calcio del material, que actúa como barrera contra el dióxido de carbono, previniendo que haya una penetración más profunda. Se cree que el límite de pH mostrado por el indicador de fenolftaleína es el resultado de la disolución de los hidratos de silicato de calcio, y la coloración rosada observada durante la prueba se debe a la alcalinidad de los hidratos de calcio. 


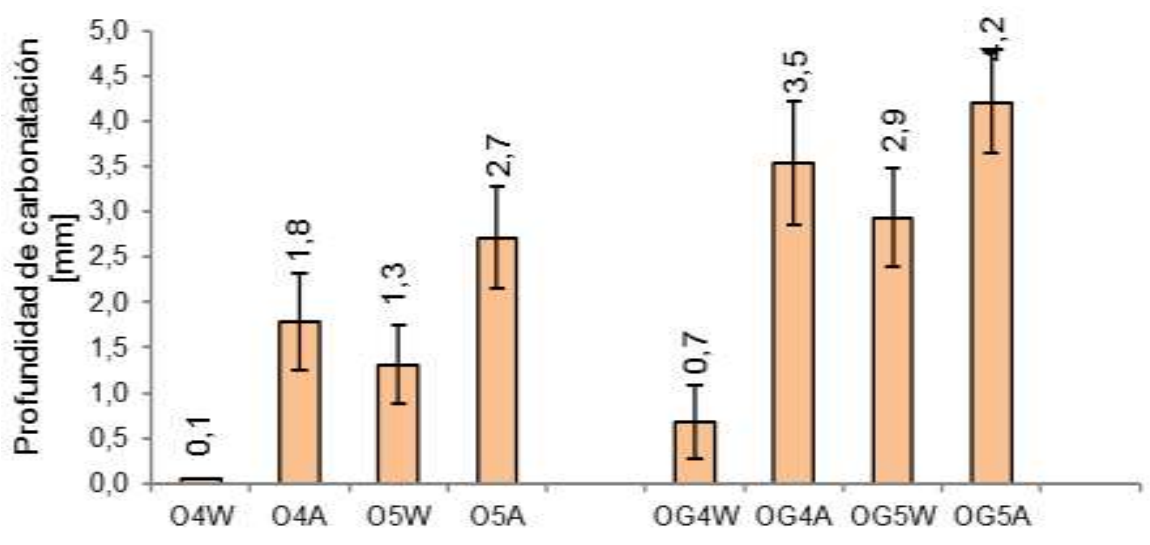

Fig. 5: Penetración del frente de Carbonatación después de 1 año de exposición en cubos de 100 mm (adaptada de Lizarazo, 2010).

Para calcular la penetración del frente de carbonatación se utilizó una solución de fenolftaleína color purpura para evaluar la reducción del pH. Las áreas sin carbonatar se observan de color purpura, mientras que las áreas carbonatadas permanecen si color. Es conocido que durante la hidratación de las mezclas CPOGGBS, la escoria no consume hidróxido de calcio, entonces, una pequeña cantidad de portlandita producida por la hidratación del cemento permanece como un barrera. Esta protección, junto con la microestructura refinada, da lugar a una adecuada resistencia a la carbonatación. Los resultados del coeficiente de carbonatación kc de la expresión (1) se muestran en la Figura 6. Se puede observar que las muestras de todas las mezclas curadas al aire presentaron un incremento importante en este coeficiente.

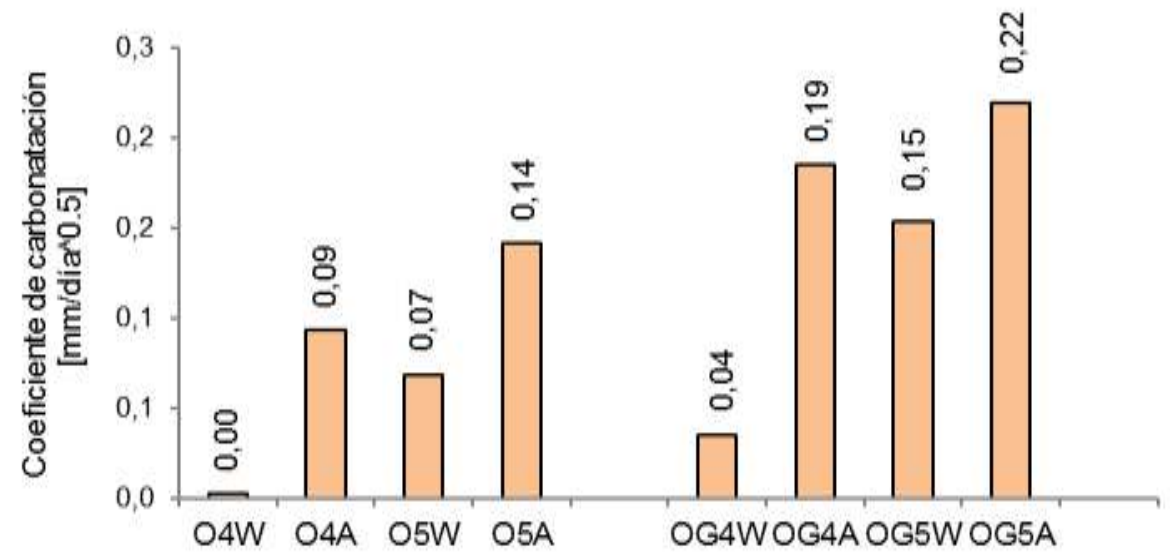

Fig. 6: Coeficiente de penetración de la Carbonación kc [mm/dias $\left.{ }^{0.5}\right]$ (adaptada de Lizarazo, 2010).

La Figura 7 muestra de forma cualitativa y a manera de ejemplo la profundidad de carbonatación de las mezclas con relaciones agua-cementante de 0.5 , pero usando probetas adicionales de sección transversal cuadrada de $50 \mathrm{~mm}$ de lado, sometidas igualmente a las condiciones de exposición descritas anteriormente durante un año.

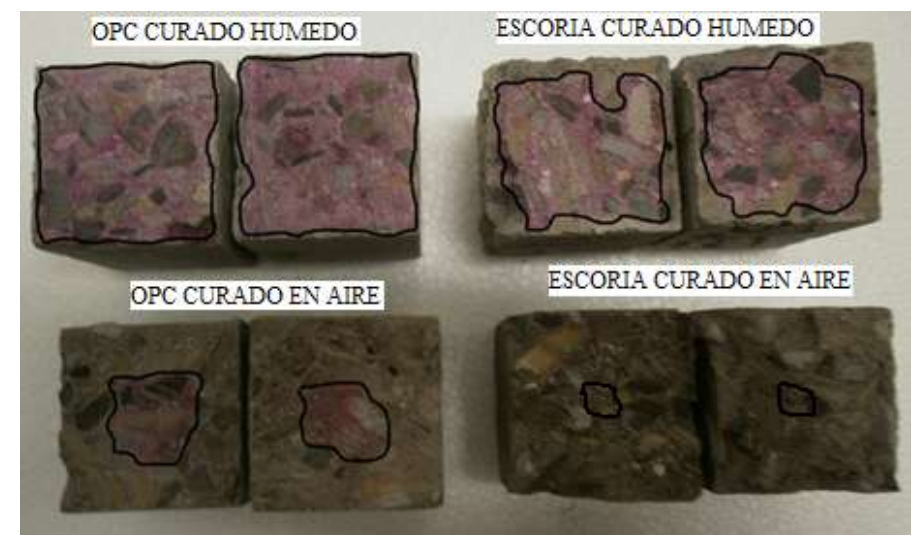

Fig. 7: Frente de carbonatación en probetas a/cm=0,5. Sección transversal cuadrada de $50 \mathrm{~mm}$ de lado(adaptada de Lizarazo, 2010) 
Penetración de lones Cloruro. La Figura 8 presenta los resultados de la penetración de iones cloruro a los 90 días de difusión. Todos los resultados se presentan con barras de error para la desviación estándar obtenida de los experimentos. La Figura 9 muestra el coeficiente de penetración de cloruros (k) calculado de la expresión (2) [mm/dias ${ }^{0.5}$. De la Figura 8 y la Figura 9 es posible observar que para las mezclas analizadas el factor más importante es la presencia de GGBS.

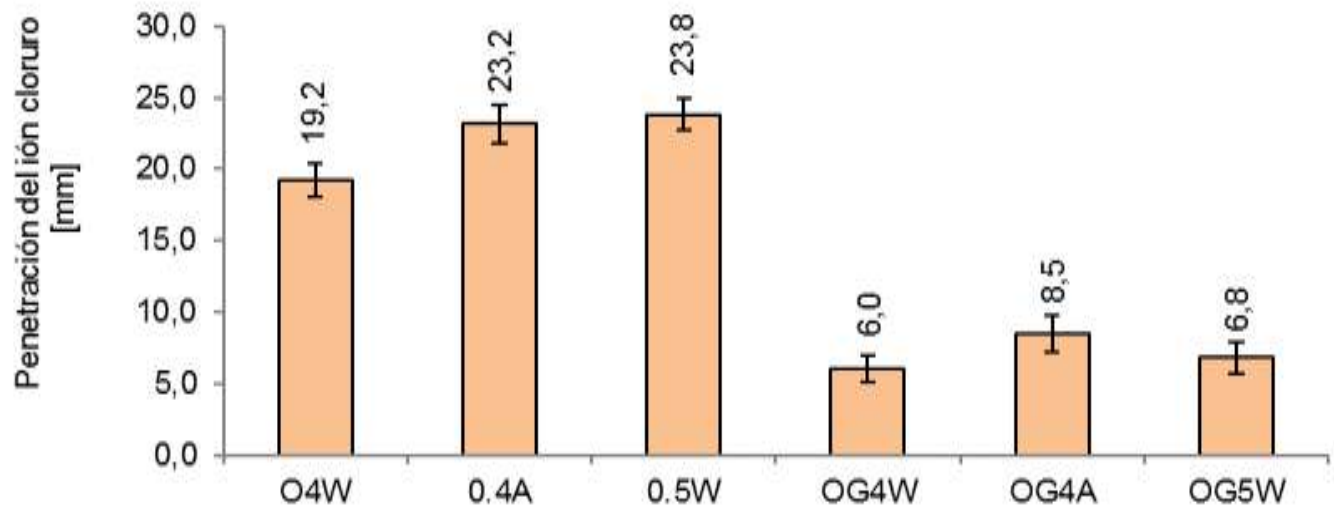

Fig. 8: Penetración Experimental de cloruros después de 90 días de auto-difusión (adaptada de Lizarazo, 2010).

Las muestras con esta adición mineral presentaron menores valores de $\mathrm{k}$ al compararlas con muestras sin esta adición. La relación agua-cementante y las condiciones de curado también influyeron en la penetración de cloruros. Para todas las mezclas, el curado al aire dio como resultado un incremento del coeficiente k, lo que podría esperarse debido a la reducción del nivel de hidratación en las capas superficiales del hormigón curado al aire. Los efectos más graves de este tipo de curado se dieron para las mezclas CPO-GGBS. Los beneficios de GGBS en la penetración de cloruros son bien conocidos y se han reportado en muchas publicaciones. En una investigación realizada por Thomas et al.(2008), Tamilarasan et al. (2012), Divsholi (2014) se encontró que la adición de escoria de alto horno en una proporción de 65\% (relación aguacemento de 0.4 ) disminuye el coeficiente aparente de difusión en una proporción de 18 con respecto a una muestra sin escoria. Las muestras CPO-GGBS curadas al agua presentaron una reducción en el coeficiente de penetración de cloruros $\mathrm{k}$ de $70 \%$ con respecto a la muestra de referencia CPO. Se cree que este buen comportamiento en términos de penetración de cloruros se debe a la capacidad de esas mezclas de fijar cloruros. La escoria aumenta la capacidad de fijación química debido a que el $\mathrm{AC}_{3}$ reacciona con los cloruros libres formando hidratos de cloroaluminato de calcio insolubles (sal de Friedel).

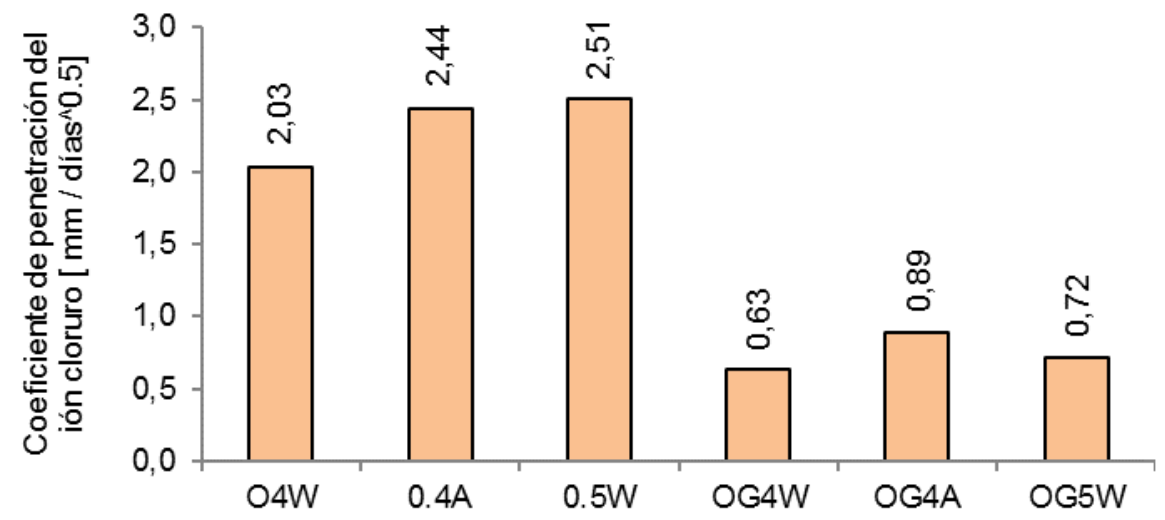

Fig. 9: Coeficientes de penetración de cloruros k calculados usando la expresión (2) (adaptada de Lizarazo, 2010).

Cambios microestructurales debidos al Curado. La Figura 10 muestra micrografías ópticas de muestras con relación agua-cementante de 0,4 que se prepararon para observarse bajo un estereoscopio y analizar los cambios en su microestructura. Las muestras se cortaron de los especímenes se pulieron ligeramente. La debilidad de las muestras curadas al aire quedó en evidencia durante la preparación de las mismas debido a la degradación de la pasta. El curado húmedo evita un secado excesivo y proporciona suficiente contenido de agua al concreto con el fin de hidratar sus capas externas. En contraste, el curado al aire incrementa el volumen de poros permeables en comparación con el curado húmedo como se demostró en las pruebas de penetración de cloruro y de carbonatación. 


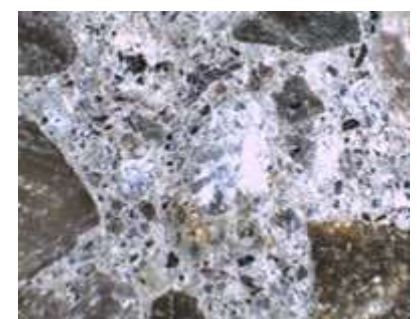

CPO CURADO HUMEDO

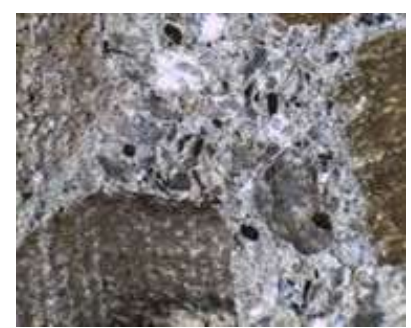

ESCORIA CURADO HUMEDO

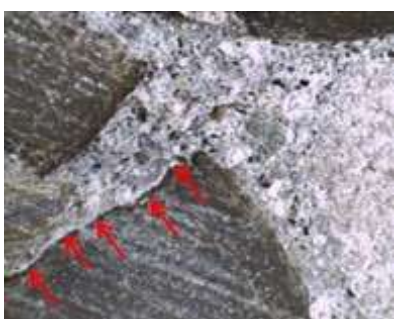

CPO CURADO EN AIRE

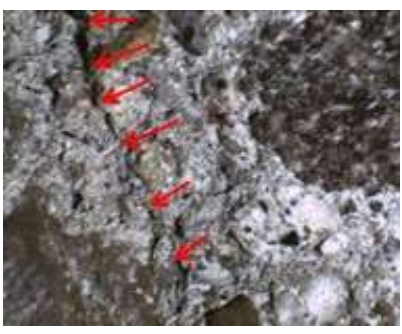

ESCORIA CURADO EN AIRE

Fig. 10: Micrografías de muestras bajo diferentes condiciones de curado

La micrografía de la muestra CPO CURADA AL AIRE muestra una delgada diferencia de nivel en la interfaz entre el agregado y la matriz de cemento, que parece ser más débil que las muestras curadas en agua. De la misma manera, esta condición se aprecia en la muestra de escoria, que también muestra de microfisuración y fisuración en la pasta. Como se observa en la Figura 8, la penetración de cloruros experimental después de 90 días de auto-difusión muestra que, cuando se añade GGBS en hormigón, estas muestras exhibieron el contenido más bajo en la penetración total de cloruros, que podría atribuirse a una mayor capacidad de fijación de cloruros, lo cual es un factor determinante para una mayor resistencia a la penetración de cloruros. De acuerdo con los resultados anteriores, se puede deducir que GGBS reestructura los poros en el hormigón debido a los productos puzolánicos que disminuyen la difusividad intrínseca, lo que confirma la eficiencia de GGBS en la prevención del daño que ocasionan los iones cloruro en los hormigones, como lo mencionan Pavía et al. (2008).

Como se ha mencionado por algunos autores (Gruyaert et al., 2013, Sulapha et al., 2003), los hormigones de escoria de alto horno son más vulnerables a la carbonatación que los hormigones de cemento Portland ordinario, debido principalmente a su menor contenido de $\mathrm{Ca}(\mathrm{OH})_{2}$. La profundidad de carbonatación también está influenciada por la porosidad del mortero en el hormigón y la presencia de agua en el mortero (Divsholi et al, 2014). Estos procesos incluyen la difusión de $\mathrm{CO}_{2}$ en fase gaseosa en los poros del hormigón, la disolución de $\mathrm{CH}$ sólido en la película acuosa de estos poros, y su reacción con el $\mathrm{CO}_{2}$ disuelto, como mencionan McPolin et al., (2009). La velocidad de difusión a la que los iones o moléculas se transportan dentro del concreto depende del tamaño y la conectividad del sistema de poros, cuando se utiliza curado húmedo en vez de curado en seco, el hormigón desarrollará un sistema de poros más refinado y menos conectado, lo que conducirá a muy bajas velocidades de transporte, de otra mano, el ingreso de $\mathrm{CO}_{2}$ se facilita y el proceso de carbonatación inicia (Papadakis, 2000). Van Balen (2005) indica que la difusividad de dióxido de carbono en el mortero es mucho mayor en el aire que en el agua. Por lo tanto una estructura de poros en el mortero que permita la transmisión de vapor es importante. Una vez en el mortero, el dióxido de carbono debe convertirse en soluble en agua para que se produzca la reacción de carbonatación. La presencia de agua en el mortero depende de las condiciones atmosféricas, la capacidad del mortero para secarse y la velocidad de las reacciones que implican la liberación y el uso del agua en el mortero. La figura indica que la tasa de carbonatación disminuyó a medida que la concentración de cemento en los morteros aumentó. Jacob (1989) reportó que a medida que la concentración de cemento aumenta la permeabilidad al vapor de los morteros disminuye. El vapor de agua y dióxido de carbono deben penetrar en el mortero para que se produzca la reacción de carbonatación. Tal comportamiento sugiere que el uso de tiempos de curado más largos dan como resultado mejoras significativas en la durabilidad.

\section{CONCLUSIONES}

De lo expuesto en este trabajo, los resultados y su discusión, se pueden extraer las siguientes conclusiones principales: (1) Todas las mezclas de escoria presentaron una evolución similar en la resistencia a la compresión que las mezclas de referencia CPO: el envejecimiento produce un aumento; el curado al aire produjo una reducción; y un incremento en la relación agua-cementante también produjo una disminución en la resistencia a la compresión; (2) La reducción en la resistencia a la compresión debido a las 
deficiencias de curado fue más significativa para mezclas que contenían CPO-GGBS. De la misma manera, las muestras CPO-GGBS curadas adecuadamente presentaron mejores resistencias que las de referencia CPO a 90 días; (3) La baja penetración de cloruros en las mezclas GGBS se atribuye a muchos factores; Sin embargo, se cree que el factor de fijación de cloruros es el predominante; (4) Debido a su reserva alcalina, el perfil de carbonatación de las muestras CPO fue muy bajo. Las muestras con GGBS presentaron mayor carbonatación que CPO y son muy vulnerables al curado deficiente; (5) La velocidad de carbonatación fue en general más rápido con el curado al aire libre de las muestras en comparación con un ambiente controlado en el laboratorio; (6) A medida que aumentaba la concentración de cemento, la velocidad de carbonatación disminuyó, según mostraron las pruebas de fenolftaleína; (7) El uso de SMC como adición a mezclas de hormigón incrementa significativamente el periodo de inicio de la corrosión inducida por cloruros; y (8) El uso de estos materiales también incrementa el tiempo de inicio a la corrosión inducida por carbonatación. Sin embargo, el uso de SCM como reemplazo de cemento Portland puede dar como resultado una disminución en el periodo de inicio de carbonatación inducida y por lo tanto aumentar el riesgo de corrosión, como mencionan Aperador et al (2013).

\section{AGRADECIMIENTOS}

El presente trabajo fue apoyado por la Universidad Nacional de Colombia - Bogotá, Proyecto DIB- 16046.

\section{REFERENCIAS}

Al-Gahtani, A.S., Effect of curing methods on the properties of plain and blended cement concretes, doi:10.1016/j.conbuildmat.2009.08.036, Const. Build. Mater., 24(3), 308-314, (2010).

Aperador W., Bautista-Ruiz J., Delgado A., Monitoreo de la Corrosión de Aceros Embebidos en Concretos obtenidos a partir de Subproductos Industriales, doi: 10.4067/S0718-07642013000400007, Inf. Tecnol. (en línea), 24(4), 55-66, (2013).

ASTM C33/C33M, Standard Specification for Concrete Aggregates, ASTM International, (2013).

ASTM C192/C192M, Standard Practice for Making and Curing Concrete Test Specimens in the Laboratory, ASTM International, (2012).

Baroghel, V., Belin, P., Maultzsch, M., Henry, D., AgNO3 spray tests: advantages, weaknesses, and various applications to quantify chloride ingress into concrete. Part 1: Non-steady-state diffusion tests and exposure to natural conditions, doi:10.1617/s11527-007-9233-1, Mater. Struct., 40, 759-781, (2007).

Basheer, P., Permeation Analysis, In Ramachandran, V. S. \& Beaudoin, J. J. (Eds.), Handbook of Analytical Techniques in Concrete Science and Technology, Noyes Publications, (2001).

BS EN 15167-1, Ground granulated blast furnace slag for use in concrete, mortar and grout. Definitions, specifications and conformity criteria, British Standard, Londres, (2006)

BS EN 197-1:, Cement. Composition, specifications and conformity criteria for common cements, British Standard, Londres, (2011)

Çakır, Ö. y Aköz, F., Effect of curing conditions on the mortars with and without GGBFS, doi:10.1016/j.conbuildmat.2006.08.013, Const. Build. Mater., 22(3), 308-314, (2008).

Chatterjee, A., X-Ray Diffraction, Handbook of Analytical Techniques in Concrete Science and Technology. Noyes Publications, (2001).

Chidiac, S. y Panesar, D.K., Evolution of mechanical properties of concrete containing ground granulated blast furnace slag and effects on the scaling resistance test at 28 days, doi:10.1016/j.cemconcomp.2007.09.003, Cem. Con. Comp., 30(2), 63-71, (2008).

Elkhadiri, I., Palacios, M. y Puertas, F. Effect of Curing Temperature on Cement Hydration, Ceramics Silikáty, 53(2), 65-75, (2009).

Divsholi B., Lim Tze Y., Teng S., Durability Properties and Microstructure of Ground Granulated Blast Furnace Slag Cement Concrete, doi 10.1007/s40069-013-0063-y, Int. J. Con. Struct. Mater., 8 (2), 157-164, (2014).

Escalante, J.I, Gómez, L.Y, Johal, K.K, Mendoza G., Mancha H., Méndez, J., Reactivity of blast-furnace slag in Portland cement blends hydrated under different conditions, doi: 10.1016/S0008-8846(01)00587-7, Cem. Con. Res., 31 (10), 1403-1409, (2001). 
Gaud, D., Rathod, H., Sharma, N., A Review on Potential of Utilizing Metal Industry Wastes in Construction Industry, Int. J. Sci. Res. Dev., 1(9), 2321-0613, (2013).

Gruyaert, E., Van den Heede, P. y De Belie, N., Carbonation of Slag Concrete: Effect of the Cement Replacement Level and Curing on the Carbonation Coefficient - Effect of Carbonation on the Pore Structure. doi:10.1016/j.cemconcomp.2012.08.024, Cem. Con. Comp., 35(1), 39-48, (2013).

Hadjsadok, A., Kenai, S., Courard, L., Michel, F., Khatib, J., Durability of mortar and concretes containing slag with low hydraulic activity, doi10.1016/j.cemconcomp.2012.02.011, Cem. Con. Comp., 34(5), 671-677, (2012).

Jacob, J. y Weiss, N. Laboratory Measurement of Water Vapor Transmission Rates of Masonry Mortars and Paints. doi: 10.2307/1504297, APT Bulletin, 21 (3-4), 62-70, (1989).

Jia Y, Aruhan B., Yan P., Natural and accelerated carbonation of concrete containing fly ash and GGBS after different initial curing period, Mag. Con. Res., 64 (2), 143-150, (2012).

Lee, Seung-Tae. Effects of curing procedures on the strength and permeability of cementitious composites incorporating GGBFS, J. Cer. Proc. Res., 9 (4), 358-361, (2008).

Lizarazo-Marriaga J., Transport properties and multi-species modelling of slag based concretes. PhD Thesis, Coventry University. Inglaterra, (2010).

McPolin, D., Basheer, P., Long, A., Carbonation and $\mathrm{pH}$ in Mortars Manufactured with Supplementary Cementitious Materials, doi 10.1061/(ASCE)0899-1561(2009)21:5(217), J. Mat. Civ. Eng., 21(5), 217-225, (2009).

Nassif, H., Najm, H., Suksawang, N., Effect of pozzolanic materials and curing methods on the elastic modulus of HPC, doi:10.1016/j.cemconcomp.2004.12.005, Cem. Con. Comp., 27(6), 661-670, (2005).

Papadakis, V., Effect of supplementary cementing materials on concrete resistance against carbonation and chloride ingress, doi:10.1016/S0008-8846(99)002495, Cem. Con. Res., 30(2), 291-299, (2000).

Pavía S. and Condren E., Study of the Durability of OPC versus GGBS Concrete on Exposure to Silage Effluent, J. Mater. Civ. Eng., 20 (4), 313-320, (2008).

Qingtao, L., Zhuguo, L., Guanglin, Y., Effects of elevated temperatures on properties of concrete containing ground granulated blast furnace slag as cementitious material, doi 10.1016/j.conbuildmat.2012.04.103, Const. Build. Mater., 35, 687-692, (2012).

Sulapha, P., Wong, S., Wee, T., Swaddiwudhipong, S. Carbonation of Concrete Containing Mineral Admixtures. doi:10.1061/(ASCE)0899-1561(2003)15:2(134), J. Mater. Civ. Eng., 134-143, (2003).

Tamilarasan V., Perumal P., Maheswaran J., Experimental Study on Water Permeability and Chloride Permeability of Concrete with GGBS as A Replacement Material for Cement, Int. J. Civ. Eng., 3 (2), (2012).

TC56 MHM-RILEM / CPC-18. Measurement of hardened concrete carbonation depth, Materials and Structures, 21(6), 453-455, (1988).

TAYLOR, H F W. Cement chemistry, $2^{\underline{a}}$ edición, 475 pp., Academic Press Limited, Londres, Inglaterra, (1990).

Thomas, M., Scott, A., Bremner, T., Bilodeau, A. y Day, D., Performance of Slag Concrete in Marine Environment, ACI Mat. J., 105(6), 628-634, (2008).

Van Balen, K., Carbonation reaction of lime, kinetics at ambient temperature, doi:10.1016/j.cemconres.2004.06.020, Cem. Con. Res., 35(4), 647-657, (2005).

Yazıcı, H., Yardımcı, M., Yigiter, H., Aydın, S. y Türkel, S., Mechanical properties of reactive powder concrete containing high volumes of ground granulated blast furnace slag, doi:10.1016/j.cemconcomp.2010.07.005, Cem. Con. Comp., 32(8), 639-648, (2010). 